\title{
Firearm Injury Encounters in the Veterans Health Administration (VHA), 2010-2015
}

\author{
Cynthia A. Lucero-Obusan*1, Aaron M. Wendelboe ${ }^{1,2}$, Patricia Schirmer ${ }^{1}$, Gina Oda ${ }^{1}$ and \\ Mark Holodniy ${ }^{1,3}$
}

'Public Health Surveillance and Research, Department of Veterans Affairs, Palo Alto, CA, USA; 2 University of Oklahoma Health Sciences, Oklahoma City, OK, USA; ${ }^{3}$ Stanford University, Stanford, CA, USA

\section{Introduction}

Firearm violence is an issue of public health concern leading to more than 30,000 deaths and 80,000 nonfatal injuries in the United States annually. ${ }^{1}$ To date, firearm-related studies among Veterans have focused primarily on suicide and attempted suicide. ${ }^{2-5}$ Herein, we examine firearm violence among VHA enrollees for all manners/ intents, including assault, unintentional, self-inflicted, undetermined and other firearm-related injury encounters in both the inpatient and outpatient settings.

\section{Methods}

Inpatient and Outpatient encounters with one or more ICD9-CM firearm external-cause-of-injury codes (E-codes) from $1 / 1 / 2010-9 / 30 / 2015$ were extracted from the VHA's Praedico ${ }^{\mathrm{TM}}$ Public Health Surveillance System, including demographics, era of service/eligibility, encounter type, and deaths. Firearm E-codes were classified for manner/intent based on the CDC's Web-based Injury Statistics Query and Reporting System (WISQARSTM) matrix. ${ }^{6}$ Outpatient/emergency department (ED) data were exclusively from VHA facilities (a single pediatric patient seen as a humanitarian emergency was excluded from the dataset). Inpatient data included VHA facilities and some records received from non-VHA facilities. VHA rate of hospitalization for firearm-related admissions was calculated using the total VHA acute-care admissions for the same time period as the denominator.

\section{Results}

During the time frame examined, 5,205 unique individuals were seen with a firearm E-code. Of these, 4,221 were seen in the outpatient/ ED setting only, 597 in the inpatient setting only, and the remaining 387 had encounters in both the outpatient/ED and inpatient settings. VHA firearm admission rate was 1.63 per 10,000 VHA admissions, compared to a national rate of 1.96 per 10,000 in 2010.7 Table 1 shows the breakdown of encounters by manner/intent. Unintentional was the most common firearm injury manner/intent. Overall, the median age at initial encounter was 54 (range 19-100 years), and $96 \%$ were male. The highest percentage served in the Persian Gulf War Era (2,136, 41\%), followed by Vietnam Era $(1,816,35 \%)$ and Post-Vietnam Era (716, $14 \%)$. The greatest number of patients with a firearm-coded encounter resided in Texas (453), California (349), Florida (326), Arizona (214) and Ohio (212).

\section{Conclusions}

Unintentional injuries were the most common form of firearm injury among VHA enrollees, representing over half of all outpatient/ED firearm encounters and more than twice the number of firearm hospitalizations compared with any other manner/intent. Limitations include that not all U.S. Veterans are VHA enrollees; miscoding and misclassification of firearm-related injuries may have occurred; and data from non-VHA outpatient/ED encounters and some non-VHA hospitalizations are not available to our surveillance system for analysis. Additional study is needed to further understand the epidemiology of firearm-related injuries among Veterans and inform VHA leadership and providers.

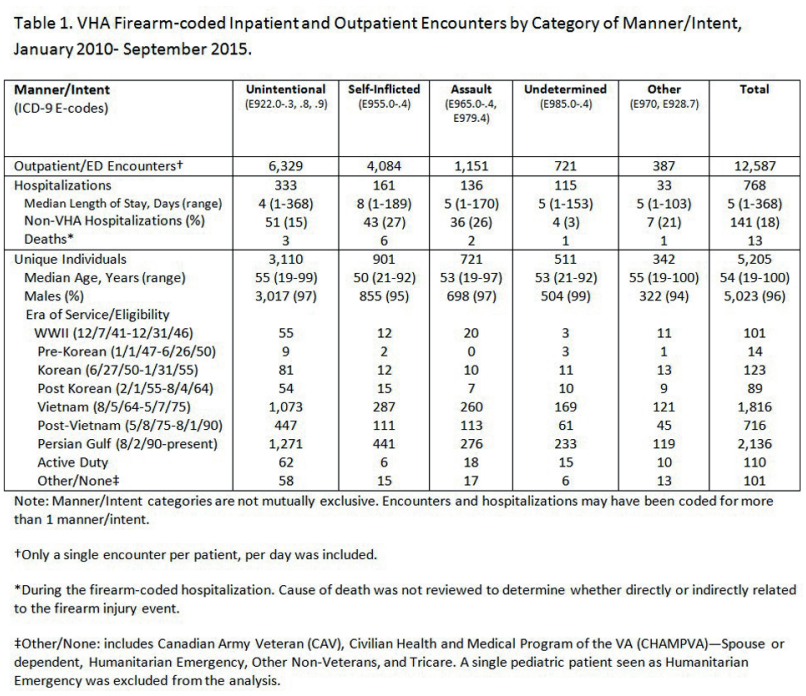

\section{Keywords}

Veterans; Firearms; Injury; Surveillance

\section{References}

1. Centers for Disease Control and Prevention. Injury Prevention and Control: Data \& Statistics (WISQARS). Available at: http://www.cdc. gov/injury/wisqars/index.html

2. McCarten JM, Hoffmire CA, Bossarte RM. Changes in Overall and Firearm Veteran Suicide Rates by Gender, 2001-2010. Am J Prev Med. 2015;48(3):360-4.

3. Kaplan MS, McFarland BH, Huguet N. Firearm Suicide Among Veterans in the General Population: Findings From the National Violent Death Reporting System. J Trauma. 2009;67:503-7.

4. Smith PN, Currier J, Drescher K. Firearm Ownership in Veterans Entering Residential PTSD Treatment: Associations with suicide ideation, attempts, and combat exposure. J. Psychiart. Res. 2015;229:220-4.

5. Walters H, Kulkarni M, Forman J, Roeder K, Travis J, Valenstein M. Feasibility and Acceptability of Interventions to Delay Gun Access in VA Mental Health Settings. J. Gen Hosp Psych. 2012;34:692-8.

6. CDC, National Center for Injury Prevention \& Control: Data \& Statistics (WISQARS), Matrix of E-code Groupings, 2011. Available at: http://www.cdc.gov/injury/wisqars/ecode_matrix.html

7. Lee J, Quraishi SA, Bhatnagar S, Zafonte RD, Masiakos PT. The Economic Cost of Firearm-related Injuries in the United States from 2006-2010. Surgery. 2014;894-8.

\section{*Cynthia A. Lucero-Obusan}

E-mail: cynthia.lucero@va.gov 